\title{
Proteomic analysis of the cerebrospinal fluid in multiple sclerosis and neuromyelitis optica patients
}

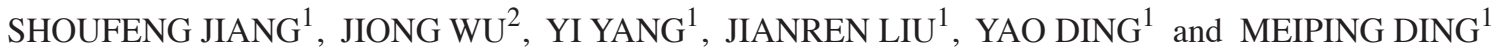 \\ ${ }^{1}$ Department of Neurology, the Second Affiliated Hospital, School of Medicine, Zhejiang University, Hangzhou, \\ Zhejiang 310009; ${ }^{2}$ Department of Neurology, Zhejiang Hospital, Hangzhou, Zhejiang 310013, P.R. China
}

Received February 29, 2012; Accepted July 25, 2012

DOI: $10.3892 / \mathrm{mmr} .2012 .1025$

\begin{abstract}
The present study aimed to compare the 2-dimensional (2D) electrophoresis pattern of the cerebrospinal fluid (CSF) in multiple sclerosis (MS), neuromyelitis optica (NMO) and control individuals, to identify the proteins with differential expression and to examine their significance. CSF samples from the three groups were collected and total protein was isolated and quantified using the Bradford method. 2D electrophoresis of the samples was conducted using equal amounts of CSF. In the CSF 2D gel electrophoresis map, 118 points were obtained from the MS group, 155 points from the NMO group and 350 points from the normal control group. Non-matching proteins appeared in 14 spots in the MS group and in 45 spots in the NMO group, and were also expressed in the $2 \mathrm{D}$ electrophoresis pattern of the normal control group. Four differential proteins were identified through the HD-MS/MS and MASCOT network search. Pre-albumin (PA) was found only in the CSF 2D gel electrophoresis map of the MS patients. Keratin 1 was expressed in the normal control group, but not in the MS group. The difference in the expression of keratin 9 in the MS group was twice that in the normal control group. The expression of keratin 1, keratin 9 and transferrin in the NMO group was twice that in the normal control group. The expression of PA was found only in the CSF 2D gel electrophoresis map of the MS patients, and not in the NMO group. Keratin 1 was expressed in the NMO group, but not in the MS group. The expression of a variety of proteins in the 2D electrophoresis pattern of CSF was significantly different in the MS, NMO and control groups. PA, keratin 1, transferrin and keratin 9 are identified as significantly differentially expressed proteins.
\end{abstract}

Correspondence to: Dr Meiping Ding, Department of Neurology, the Second Affiliated Hospital, School of Medicine, Zhejiang University, No. 88 Jiefang Road, Hangzhou, Zhejiang 310009, P.R. China E-mail: sfj2001@yeah.net

Key words: multiple sclerosis, neuromyelitis optica, proteomics, cerebrospinal fluid

\section{Introduction}

Multiple sclerosis (MS) is an autoimmune inflammatory disease of the central nervous system. The demyelination process is characterized by an inter-, not an intra-individual, heterogeneity. Four distinct patterns of focal demyelination have been identified histologically in biopsy and autopsy materials. Neuromyelitis optica (NMO) is an idiopathic inflammatory demyelinating disease of the central nervous system, affecting predominantly the optic nerves and the spinal cord (1). NMO is a severe monophasic syndrome characterized by bilateral optic neuritis and myelitis occurring in rapid succession. Serum NMO-immunoglobulin G (IgG) has been investigated, and its presence is highly specific for NMO $(2,3)$. However, NMO-IgG is not detectable in all patients. Therefore, NMO diagnosis is generally based on a combination of clinical, neuroimaging, laboratory and pathological findings $(4,5)$.

Although both MS and NMO are demyelinating diseases of the central nervous system, they differ in a variety of ways, such as in pathogenesis, pathology, clinical features and treatment options. Cerebrospinal fluid (CSF) proteomics is a well-accepted powerful technique to monitor protein expression in CSF. Abnormal CSF findings are one of the characteristics of NMO or MS, and may provide important information to decipher NMO mechanisms or MS pathogenesis. In the present study, the differences in the CSF protein of an MS group, an NMO group and a normal control group of individuals were compared using 2D gels. The aim of the study was to find significant potential protein disease markers and to discover disease pathogenesis. Understanding the expression and functions of these disease-related proteins may enable us to identify them as possible biomarkers to aid in the diagnosis of NMO and, possibly, in its treatment and prognosis as well.

\section{Materials and methods}

Participants and preparation. The selected patients matched the 2005 version of the McDonald diagnostic criteria for MS. The patients were all in the recurrent-remitting (R-R) acute period with the following characteristics: male/female (2/4), age

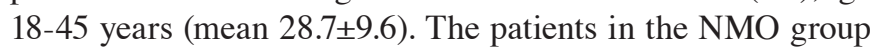
matched the Wingerchuk diagnostic criteria for NMO with the 
following characteristics: male/female (3/3), age 21-39 years (mean 27.2 \pm 8.4 ). The control group consisted of healthy subjects with the following characteristics: male/female (3/3), age 19-35 years (mean 25.2 \pm 6.2 ). The subjects in the control group did not suffer from any specific previous disorders and their neurological examinations showed no pathological signs. The study was approved by the ethics committee of the Second Affiliated Hospital, School of Medicine, Zhejiang University.

With the consent of the volunteers, CSF samples were collected by lumbar puncture. Following the routine clinical tests for CSF, the remaining samples were placed in sterile centrifuge tubes and centrifuged for $15 \mathrm{~min}$ at a speed of $2,000 \mathrm{rpm}$. Following centrifugation, the supernatant fluid was preserved and distributed in sterile EP tubes. The EP tubes were then placed in a refrigerator at $-80^{\circ} \mathrm{C}$ for later use. The lumbar puncture procedure was performed at normal pressure. The results of the routine CSF and biochemical tests are shown in Table I.

Protein purification. The CSF samples were mixed and distributed in Amicon Ultra-15 centrifugal ultra-filtration tubes. A high-speed low-temperature centrifuge was placed at a fixed angle of $35^{\circ}$. The centrifugation lasted for $45 \mathrm{~min}$ at $4{ }^{\circ} \mathrm{C}$ and $5,000 \mathrm{x} \mathrm{g}$. Then, the concentrate $(\sim 200 \mu \mathrm{l})$ was collected.

Following the ProteoExtract Albumin/IgG Removal kit affinity chromatography instructions, the original retained buffering solution of the chromatograph was removed. The chromatograph was washed with $0.7 \mathrm{ml}$ specific buffering solution. The buffering solution descended through the resin by gravity. The diluted CSF was added to the chromatography column. The cerebrospinal fluid was allowed to descend through the resin bed by gravity, and the eluate was saved.

$2 D$ electrophoresis. The first phase of the isoelectric focusing electrophoresis was conducted using the IPGphor IEF System. The protein was centrifuged for $2 \mathrm{~min}$ before the sample was loaded. Then, $100 \mu \mathrm{g}$ of the sample was loaded and resolved with a rehydration solution containing $8 \mathrm{M}$ urea, $0.02 \%$ CHAPS, $0.02 \mathrm{M}$ DTT and $0.05 \%$ IPG buffering solution. Approximately $800 \mu \mathrm{l}$ of covering fluid was added. The focused strip was balanced twice on the SDS balance solution (1.5 M tris-Cl, pH 8.8, $50 \mathrm{mM}, 30 \%$ Glycerol, $6 \mathrm{M}$ urea, $2 \%$ SDS and Bromophenol blue trace) and then shaken twice on the oscillation bed for $15 \mathrm{~min}$. MDTT $(20 \mathrm{mM})$ was added to the first balance solution, whereas $100 \mathrm{mM}$ of iodoacetamide was added to the second balance solution. The strip was then moved to the PROTEAN II xi Cell to be processed in the vertical SDS-PAGE for the second phase. A 13\% polyacrylamide gel separator was used with a constant current of $40 \mathrm{~mA}$ for $40 \mathrm{~min}$ and $60 \mathrm{~mA}$ for $5 \mathrm{~h}$. The process continued until the front of the bromophenol blue reached the bottom of the glass. This electrophoresis procedure was repeated three times for each group of samples (Table II).

Scanning and image analysis. The gel was scanned by an image scanner and the images were saved in TIFF format. The scanned images were analyzed using the ImageMaster 2D Platinum5.0 software. The test parameters, particularly the smoothness optimization parameters, were set to detect all the real protein spots and to split the overlapping protein
Table I. Comparison of the CSF tests.

\begin{tabular}{lccc}
\hline & MS & NMO & Control \\
\hline Gender (M/F) & $2 / 4$ & $3 / 3$ & $3 / 3$ \\
Age (years) & $28.7 \pm 9.6$ & $27.2 \pm 8.4$ & $25.2 \pm 6.2$ \\
Pressure (mmHg) & $156.7 \pm 19.4$ & $148.6 \pm 23.5$ & $129.3 \pm 12.8$ \\
Leukocyte (l) & $20.2 \pm 8.6$ & $18.4 \pm 6.4$ & $1.2 \pm 0.8$ \\
Protein $(\mathrm{mg} / \mathrm{l})$ & $457.9 \pm 42.2$ & $359.4 \pm 32.1$ & $355.7 \pm 30.9$ \\
Chloride $(\mathrm{mmol} / \mathrm{l})$ & $129.1 \pm 33.5$ & $124.4 \pm 29.7$ & $125.4 \pm 21.5$ \\
Glucose (mmol/l) & $3.6 \pm 2.1$ & $4.1 \pm 2.9$ & $3.0 \pm 1.5$ \\
\hline
\end{tabular}

MS, multiple sclerosis; NMO, neuromyelitis optica.

Table II. Silver staining.

Steps

Time (min)

1. Stationary liquid

30

2. Sensitizer

30

3. Washing

$3 \times 5$

4. Silver reaction

20

5. Washing

$2 \times 1$

6. Staining

$2-5$

7. Terminator

10

spots. By adjusting the saliency and minimum area values, the noise interference was filtered. Thus, automatic detection (spot detection) was completed and quantified and the background was removed. The manual use of the spot-marking method enables the increase or removal of the number of spots, and copies and splits the spots, thus gaining access to the protein spot detection map. In the present study, the molecular weight (MW) values of the protein spots were obtained by marking the marker protein and its $\mathrm{pH}$ value. Finally, a reference gel (refelenee gel) was selected for comparison to other gels and a gel analysis report was generated. The total volume percentage of one specific spot to the gel was computed, and the differences in protein spots were compared using statistical analysis (between group t-tests). Finally, the protein spots were differentiated from the standard to determine whether the analytical difference was significant and whether the volume percentage was twice as large.

Preparation of mass spectrometry sample. The tips of $200 \mu \mathrm{l}$ yellow pipettors were cut into appropriate sizes (preferably of the same size) and immersed in washing lotion for 1 day. Distilled water and re-distilled water were used to immerse them sequentially, and they were then processed with ultrasonic waves. The gel samples were washed thoroughly with re-distilled water and baked to dry. The protein of interest was extracted from the gel with the cut gun tips or cut with a $1.5-\mathrm{mm}$ gel pen and placed in EP tubes or 96-hole boards. The corresponding numbers of the spots and their positions were recorded. 
The gel was washed twice with $50 \mu 1$ DDW for $10 \mathrm{~min}$ each time. Up to $50 \mu 1$ acetonitrile was added to dehydrate the gel until it turned completely white. The mixture was pumped into a vacuum for $5 \mathrm{~min}$. Then, $20 \mu \mathrm{l}$ of $10 \mathrm{mM}$ DTT (prepared with $10 \mu \mathrm{l} 1 \mathrm{M}$ DTT, $990 \mu 125 \mathrm{mM} \mathrm{NH}_{4} \mathrm{HCO}_{3}$ ) was added. The mixture was bathed in $56^{\circ} \mathrm{C}$ water for $1 \mathrm{~h}$. After cooling to room temperature, the gel was dried and $20 \mu \mathrm{l}$ of $55 \mathrm{mM}$ IAA (prepared with $55 \mu 11 \mathrm{M}$ IAA, $945 \mu 125 \mathrm{mM}$ $\mathrm{NH}_{4} \mathrm{HCO}_{3}$ ) was quickly added. The mixture was kept in a dark room for $45 \mathrm{~min}$. The gel particles were subsequently washed with $25 \mathrm{mM} \mathrm{NH}_{4} \mathrm{HCO}_{3}, 25 \mathrm{mM} \mathrm{NH}_{4} \mathrm{HCO}_{3}$ and $50 \%$ acetonitrile solution, and then dehydrated with acetonitrile until the mixture turned completely white. Then, it was pumped through a vacuum for $5 \mathrm{~min}$ to dry. The $0.1-\mu \mathrm{g} / 1$ stock trypsin solution was diluted 10 times with $25 \mathrm{mM}$ $\mathrm{NH}_{4} \mathrm{HCO}_{3}$, and $2 \mu \mathrm{l}$ of the dilution was added to each EP tube. Short-term centrifugation was carried out to make sure the gel particles were fully mixed with the enzyme. The EP tubes were kept in a $4^{\circ} \mathrm{C}$ environment or placed on ice for 30 min until the solution became completely absorbed by

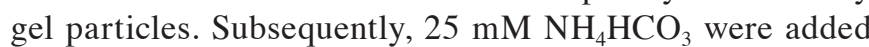
to each tube for a total volume of 10-15 $\mu$. The tubes were stored at $37^{\circ} \mathrm{C}$ to be digested overnight. The next day, $2 \%$ TFA was added to each tube to stop the reaction. The final TFA concentration was maintained at $0.1 \%$. The mixture was then shaken and centrifuged.

$H D-M S / M S$ analysis. Following digestion, the enzyme hydrolyzate sample was loaded onto a 300-hole $1-\mu$ l volume stainless steel model. The same sample loading procedure was repeated two more times after the first sample spots became dry. Then, $1 \mu \mathrm{l}$ of substance (CHCA) was added. Approximately $1.1 \mu \mathrm{l}$ of the calibration mixture was added in the middle of the substance for calibration purposes while loading the sample around it. The peak of the trypsin selfhydrolyzate was used as internal calibration. The model was left at room temperature to dry. It was then sent to the Beijing Military Medical Sciences Mass Spectrometry Laboratory for HD-MS/MS analysis using the Synapt HDMS produced by Waters Micromass Corporation.

Database search. The search was performed using MASCOT software which may be found by logging into the Protein database via the internet website (http://www.matrix-science.co.uk).

Preliminary analysis of protein spots. The features of the proteins were searched and identified from the internet protein search site (http://www.expasy.ch/swiss-2dpage/ viewer) according to their characteristics, such as their isoelectric point and relative molecular mass, which were obtained through the gel analysis report. They were then compared to the 2D-PAGE reference map of the CSF from the SWISS-2DPAGE database. The preliminary analysis of these proteins was performed in accordance with relevant literature on this subject.

\section{Results}

In the CSF 2D gel electrophoresis map, 118 points were obtained from the MS group, 155 points were obtained from

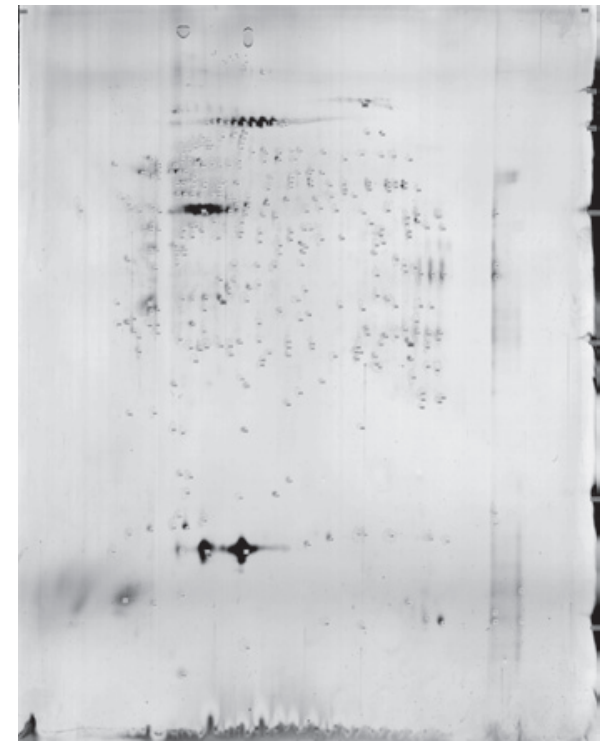

Figure 1. CSF 2D gel electrophoresis map of the normal control group (after analysis).

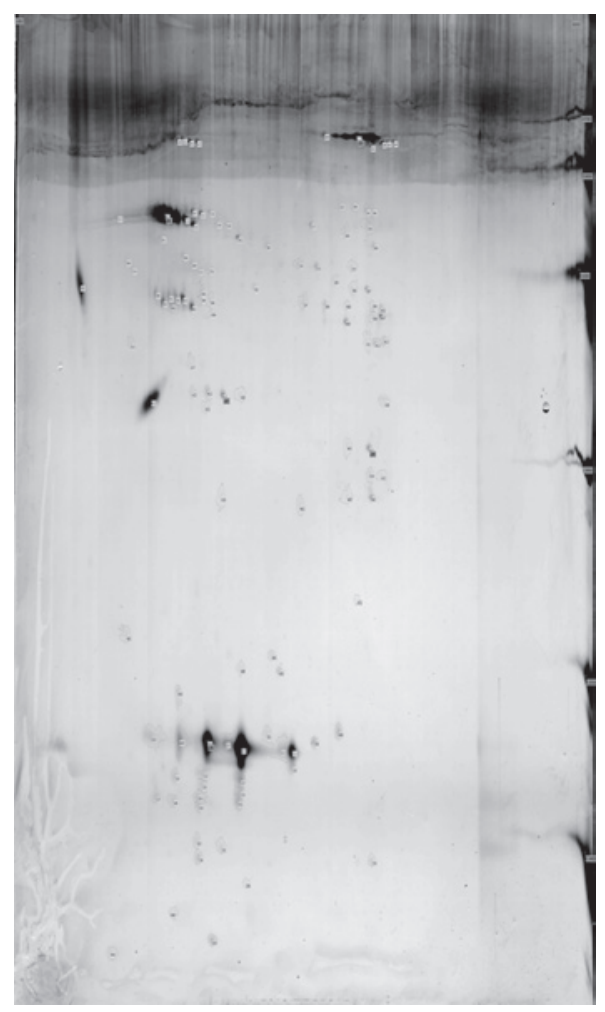

Figure 2. CSF 2D gel electrophoresis map of the MS group (after analysis).

the NMO group and 350 points were obtained from the normal control group (Figs. 1-3).

Comparing the CSF 2D gel electrophoresis pattern of the MS group and the control group, 51 matching protein spots were found. Among these matching spots, the MS group had 38 upregulated proteins, whereas the normal group had 24. Twenty-four spots had an upregulating difference of $>2$ times. On the other hand, 13 protein spots were downregulated. The number of non-matching protein spots was 67 for the MS group and 299 for the normal control group. 
Table III. Differences of CSF in protein spectrum analysis of the MS and control groups.

\begin{tabular}{lcccc}
\hline Spot & Protein name & Accession no. & Experimental mass/pI & MASCOT score \\
\hline MS511 & Pre-albumin & gil219978 & $15909 / 5.52$ & 144 \\
Control191 & Keratin 1 & gil7331218 & $65978 / 8.16$ & 184 \\
MS493 & Cytokeratin 9 & gil435476 & $62092 / 5.19$ & 149 \\
\hline
\end{tabular}

MS, multiple sclerosis.

Table IV. Differences of CSF in protein spectrum analysis of the NMO and control groups.

\begin{tabular}{lcccc}
\hline Spot & Protein name & Accession no. & Experimental mass/pI & MASCOT score \\
\hline NMO232 & Transferrin & gil4557871 & $77000 / 6.81$ & 318 \\
NMO334 & Keratin 9 & gil435476 & $62092 / 5.19$ & 149 \\
NMO161 & Keratin 1 & gil7331218 & $65978 / 8.16$ & 184 \\
\hline
\end{tabular}

NMO, neuromyelitis optica.

Table V. Differences of CSF in protein spectrum analysis of the MS and NMO groups.

\begin{tabular}{lcccc}
\hline Spot & Protein name & Accession no. & Experimental mass/pI & MASCOT score \\
\hline MS511 & Pre-albumin & gil219978 & $15909 / 5.52$ & 144 \\
NMO161 & Keratin 1 & gil7331218 & $65978 / 8.16$ & 106 \\
\hline
\end{tabular}

MS, multiple sclerosis; NMO, neuromyelitis optica.

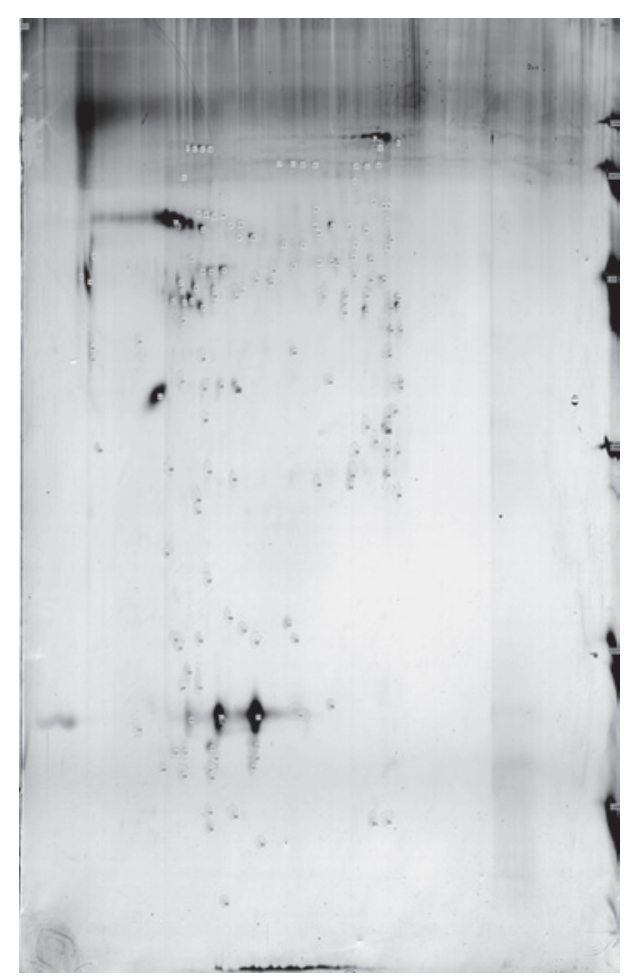

Figure 3. CSF 2D gel electrophoresis map of the NMO group (after analysis).
Comparing the CSF 2D gel electrophoresis pattern of the $\mathrm{NMO}$ and the control group, 82 matching protein spots were found. Among these matching spots, the NMO group had 64 upregulated proteins compared to the normal group, and 41 spots had $>2$ upregulated differences. On the other hand, 18 protein spots were downregulated. The non-matching protein spots were 73 for the NMO group, while for the normal control group they were 268.

There were $45 \mathrm{CSF}$ protein spots expressed in the control group, but not in the NMO group and the MS group. Conversely, 14 CSF protein spots were expressed in the control and the MS groups, but not in the NMO group.

By HD-MS/MS analysis and MASCOT web search, three differential proteins were identified, i.e., pre-albumin (PA), keratin 1 and keratin 9, and transferrin (Tf). PA was only found in the CSF 2D gel electrophoresis map of the MS patients. Keratin 1 was expressed in the normal control group, but not in the MS group. The difference in the expression of keratin 9 in the MS group was two times higher than that of the normal control group. The expression of keratin 1, keratin 9 and $\mathrm{Tf}$ in the NMO group was two times higher than that of the normal control group. The expression of PA was found in the CSF 2D gel electrophoresis map of the MS patients, but not in the NMO group. Keratin 1 was expressed in the NMO group, but not in the MS group (Tables III-V). 


\section{Discussion}

Numerous studies confirm that changes in the composition of CSF are closely related to the demyelinating diseases of the central nervous system. Thus, CSF is highly suitable material for proteomic analysis of central nervous system demyelinating diseases. However, the concentration of protein in CSF is extremely low, and albumin and $\mathrm{IgG}$ comprise $99 \%$, making the abundance of specific diseaserelated proteins very low. Thus, the highly-abundant proteins have to be removed before conducting $2 \mathrm{D}$ electrophoresis. In the present study, ProteoExtract Albumin/IgG Removal kit affinity chromatography was used to remove the highly abundant proteins. An accurate 2D gel electrophoresis map was obtained for further analysis.

Although the use of the proteomic analysis method to study CSF remains in the trial stage, studies that apply this method to MS patients have increased in recent years. Noben et al adopted the LC-ESI-MS/MS technique to compare the proteome differences of CSF in MS and non-MS groups (6). They identified a total of 148 proteins as follows: 80 co-expression proteins from the two groups, 24 proteins that were not expressed in the MS group and 44 for the non-MS group. Ottervald et al used the 2D gel electrophoresis tandem mass spectrometry technique to analyze CSF samples of MS patients and controls, and found that three proteins act as potential MS marking proteins: $\alpha 1$ anti-chymotrypsin, $\alpha 1$-macroglobulin and angiotensinogen (7). Tumani et al used 2D differential gel electrophoresis and matrix-assisted laser desorption flight mass spectrometry techniques to compare CSF samples of confirmed MS patients and patients with suspected nerve inhibition. Using enzyme-linked immunosorbent assay, they found that the level of serine peptidase inhibiting protein in the CSF of MS patients increased, whereas the amounts of $\alpha$-B-glycoprotein, fetuin A, serum apolipoprotein $\mathrm{A} 4$, haptoglobin, transferrin, ultra-oxidants superoxide dismutase I, retinol-binding protein, human zinc$\alpha$-2-glycoprotein and eight others decreased. However, more explicit evidence on how the pathophysiology of these proteins is linked to clinical diagnosis is necessary. Although many related studies are currently being conducted, due to the differences in experimental methods, purposes and subjects, as well as the complexity of proteomic analysis, current proteomic studies remain in their infancy. Furthermore, different studies often fail to give unanimous results; thus, further research is required.

CSF proteomics studies on NMO patients are relatively fewer. One relevant study is that of Bai et al (8) from the Medicine Biochemistry and Molecular Biology Institute of Shandong University, who analyzed CSF 2D electrophoresis maps and matrix-assisted laser desorption ionization-flight time mass spectrometry (MALDI-TOF-MS/MS) of NMO patients, and found that their CSF had four upregulated and seven downregulated proteins.

In the present study we developed the CSF 2D gel electrophoresis maps of MS patients, NMO patients and normal controls. We obtained further related protein information through image analysis. We noted and identified four proteins that have significant differences, i.e., PA, keratin 1, Tf and keratin 9. The keratin 9 expression in the MS group was eight times higher than that in the control group. PA was highly expressed only in the CSF 2D gel electrophoresis maps of MS patients and did not express in the control group. Keratin 1 was expressed in the normal control group, but not in the MS group. The expression of Tf, keratin 9 and keratin 1 proteins in the NMO group was four, eight and seven times higher than that of the normal control group, respectively. PA was highly expressed in the MS group, but not in the NMO group. Keratin 1 was highly expressed in the NMO group, but not in the MS group.

PA is a carrier protein in the synthesis of liver cells. It is a stable tetramer which consists of four identical subunits. Each subunit consists of 127 amino acid residues and important biological activity. They participate in plasma thyroid hormone transportation and vitamin A transportation in the blood circulation. PA derives its name from its electrophoretic mobility, which is greater than that of albumin, thus reaching its location prior to albumin. It is also known as thyroid-binding PA or vitamin A transport protein. PA can be detected in fetal blood at 11.5 weeks of pregnancy. It has a radioactive half-life period of 1.9 days and is classified under the fast-transporter proteins. PA may be affected by estrogen and it has an impact on the activity of the thymus hormone. It also increases immunity by inducing the maturation of lymphocytes. Under stressed conditions and defective energy metabolism, PA concentration declines rapidly. Hence, it is a negative acute-phase protein (9). PA includes one of the water-soluble proteins of the brain tissue. The ratios of PA in gray and in white matter are 1.9 and $1.1 \%$, respectively. PA in CSF form comes primarily from the brain tissue and a small part from plasma infiltration (10). The main source of brain PA is the choroid. Whether the high expression of PA in the CSF of patients with MS is associated with the pathogenesis of MS and how the association is developed require further exploration.

Tf includes blood (serum) Tf (serotransferrin, STf; derived from the serum of vertebrates), egg (ovalbumin) Tf (ovotransferrin, OTf; derived from bird egg and serum) and milk (whey) transferrin, which is also known as lactoferrin (lactotransferrin, LTf; derived from mammalian milk and other secretions). Tf is an important $\beta$-globulin and acts as iron transporter for the vertebrates. It is also an essential component of vertebrate humoral and cells. Tf is a single chain glycosylated protein which consists of approximately $6 \%$ glycosylation and two highly homologous structural domains at the N-terminal and $\mathrm{C}$-terminal. The main physiological function of $\mathrm{Tf}$ is to transport iron from the place it is absorbed and to store it in the erythrocyte for the synthesis of hemoglobin (11). Tf regulates iron balance and energy balance. In ion exchange kinetics, Tf not only changes the quality of materials, but also converts their energies. In addition, Tf is the key factor in the vertebrate respiratory chain (or respiratory network) that directly participates in iron transport and metabolism. The iron ions transported by Tf are delivered into the erythrocyte to be used in the synthesis of hemoglobin and play an important role in blood circulation. Tf is also involved in cell proliferation and immune system regulation and is a necessary growth factor for cell growth and proliferation. In tumor and cancer cells, the $\mathrm{Tf}$ receptor content is significantly higher than in the corresponding normal cells. Finally, Tf is a non-specific immune factor in bodies of vertebrates. It serves antibacterial and 
antiseptic purposes and is an important factor in the inhibition of bacterial growth. Hence, Tf has a relatively comprehensive protein physiological function and belongs to a special class of functional proteins $(12,13)$.

Tf in CSF comes from serum and is approximately $1 / 100$ of the serum level. A dynamic, balanced relationship exists between them (14). Przyjalkowski et al (15) posited that Tf is a good indicator for blood-brain barrier (BBB) damage. By measuring the Tf level in CSF, insight may be gained into BBB damage and the condition of the disease, which is useful in guiding the treatment. Combining this information with our study, we believe that the high expression of Tf in CSF may be associated with impaired $\mathrm{BBB}$ in patients with $\mathrm{NMO}$, and could be considered as a response indicator of severe BBB damage during the pathological NMO process.

Keratin 1 belongs to class II keratins, and is expressed in the stratum spinosms, which are on the base of the spine following mitosis. It modulates the expression of keratin 1, which belongs to class I keratins, and they are often expressed in pairs and bonded together (16). Keratin 1 and keratin 10 are associated with cell differentiation and maturation. Studies have shown that their expression can be found in squamous cell carcinoma, which comes from the skin and from the internal organs (17). For unknown reasons, keratin 1 expression does not appear in MS. The reason behind the high expression of keratin 1 in the CSF of both the normal control and the NMO groups, and its non-expression in the MS group, remain unknown.

Keratin 9 is a class I keratin mainly expressed in palmoplantar epidermis (18) and dispersedly expressed in the other areas of the epithelium (19). It is often paired with keratin 1 and reflects the function of keratin on the regulation of glial cell differentiation (20). The function of keratin 9 in the pathogenesis of MS has not been reported.

\section{References}

1. Matiello M, Jacob A, Wingerchuk DM and Weinshenker BG: Neuromyelitis optica. Curr Opin Neurol 20: 255-260, 2007.

2. Saiz A, Zuliani L, Blanco Y, Tavolato B, Giometto B and Graus F: Revised diagnostic criteria for neuromyelitis optica (NMO) Application in a series of suspected patients. J Neurol 254: 1233-1237, 2007.

3. Lennon VA, Wingerchuk DM, Kryzer TJ, et al: A serum autoantibody marker of neuromyelitis optica: distinction from multiple sclerosis. Lancet 364: 2106-2112, 2004.
4. Argyriou AA and Makris N: Neuromyelitis optica: a distinct demyelinating disease of the central nervous system. Acta Neurol Scand 118: 209-217, 2008

5. Lana-Peixoto MA: Devic's neuromyelitis optica: a critical review. Arq Neuropsiquiatr 66: 120-138, 2008.

6. Noben JP, Dumont D, Kwasnikowska N, et al: Lumbar cerebrospinal fluid proteome in multiple sclerosis: characterization by ultrafiltration, liquid chromatography, and mass spectrometry. J Proteome Res 5: 1647-1657, 2006.

7. Ottervald J, Franzen B, Nilsson K, et al: Multiple sclerosis: identification and clinical evaluation of novel CSF biomarkers. J Proteomics 73: 1117-1132, 2010.

8. Bai S, Liu S, Guo X, et al: Proteome analysis of biomarkers in the cerebrospinal fluid of neuromyelitis optica patients. Mol Vis 15: 1638-1648, 2009.

9. Svenungsson E, Gunnarsson I, Fei GZ, Lundberg IE, Klareskog L and Frostegard J: Elevated triglycerides and low levels of highdensity lipoprotein as markers of disease activity in association with up-regulation of the tumor necrosis factor alpha/tumor necrosis factor receptor system in systemic lupus erythematosus. Arthritis Rheum 48: 2533-2540, 2003

10. Henry BJ (ed): Clinical Diagnosis and Management by Laboratory Methods. 18th edition. WB Saunders Company, Philadelphia, pp451, 1991

11. Qian ZM, Li H, Sun H and Ho K: Targeted drug delivery via the transferrin receptor-mediated endocytosis pathway. Pharmacol Rev 54: 561-587, 2002.

12. Holmberg CG and Laurell CB: Investigations in serum copper; nature of serum copper and its relation to the iron-binding protein in human serum. Acta Chem Scand 1: 944-950, 1947.

13. Williams J: The evolution of transferrin. Trends Biochem Sci 7: 394-397, 1982.

14. Gruener N, Gozlan O, Goldstein T, Davis J, Besner I and Iancu TC: Iron, transferrin, and ferritin in cerebrospinal fluid of children. Clin Chem 37: 263-265, 1991.

15. Przyjalkowski W, Lipowski D, Kolasa T, Issa E and Olejnik Z: Blood-cerebrospinal fluid barrier in purulent cerebrospinal meningitis. Neurol Neurochir Pol 30: 39-48, 1996 (In Polish).

16. Stoler A, Kopan R, Duvic M and Fuchs E: Use of monospecific antisera and cRNA probes to localize the major changes in keratin expression during normal and abnormal epidermal differentiation. J Cell Biol 107: 427-446, 1988.

17. Moll R: Cytokeratins as markers of differentiation in the diagnosis of epithelial tumors. Subcell Biochem 31: 205-262, 1998.

18. Langbein L, Heid HW, Moll I and Franke WW: Molecular characterization of the body site-specific human epidermal cytokeratin 9: cDNA cloning, amino acid sequence, and tissue specificity of gene expression. Differentiation 55: 57-71, 1993.

19. Moll I, Heid H, Franke WW and Moll R: Distribution of a special subset of keratinocytes characterized by the expression of cytokeratin 9 in adult and fetal human epidermis of various body sites. Differentiation 33: 254-265, 1987.

20. Swensson O, Langbein L, McMillan JR, et al: Specialized keratin expression pattern in human ridged skin as an adaptation to high physical stress. Br J Dermatol 139: 767-775, 1998. 